\title{
Cognitive and Cortical Plasticity Deficits Correlate with Altered Amyloid- $\beta$ CSF Levels in Multiple Sclerosis
}

\author{
Francesco Mori' ${ }^{1,2}$, Silvia Rossi' ${ }^{1,2}$, Giulia Sancesario ${ }^{3,4}$, Claudia Codecà 1,2, Giorgia Mataluni ${ }^{1,2}$, Fabrizia \\ Monteleone ${ }^{1,2}$, Fabio Buttari ${ }^{1,2}$, Hajime Kusayanagi ${ }^{1,2}$, Maura Castelli ${ }^{1,2}$, Caterina Motta ${ }^{1,2}$, Valeria Studer ${ }^{1,2}$, \\ Giorgio Bernardi $^{1,2}$, Giacomo Koch ${ }^{1,2}$, Sergio Bernardini ${ }^{3,4}$ and Diego Centonze ${ }^{*, 1,2}$ \\ 'Dipartimento di Neuroscienze, Clinica Neurologica, Università Tor Vergata, Rome, Italy; ${ }^{2}$ Centro Europeo per la Ricerca sul Cervello \\ (CERC)/Fondazione Santa Lucia, Rome, Italy; ${ }^{3}$ Dipartimento di Medicina Interna, Università Tor Vergata, Rome, Italy; ${ }^{4}$ Dipartimento di Medicina \\ di Laboratorio, UOC Biologia Molecolare Clinica, Policlinico Tor Vergata, Rome, Italy
}

\begin{abstract}
Cognitive dysfunction is of frequent observation in multiple sclerosis (MS). It is associated with gray matter pathology, brain atrophy, and altered connectivity, and recent evidence showed that acute inflammation can exacerbate mental deficits independently of the primary functional system involved. In this study, we measured cerebrospinal fluid (CSF) levels of amyloid- $\beta_{1-42}$ and $\tau$ protein in MS and in clinically isolated syndrome patients, as both proteins have been associated with cognitive decline in Alzheimer's disease (AD). In AD, amyloid- $\beta_{1-42}$ accumulates in the brain as insoluble extracellular plaques, possibly explaining why soluble amyloid- $\beta_{1-42}$ is reduced in the CSF of these patients. In our sample of MS patients, amyloid- $\beta_{1-42}$ levels were significantly lower in patients cognitively impaired (Cl) and were inversely correlated with the number of Gadolinium-enhancing $(\mathrm{Gd}+)$ lesions at the magnetic resonance imaging (MRI). Positive correlations between amyloid- $\beta_{1-42}$ levels and measures of attention and concentration were also found. Furthermore, abnormal neuroplasticity of the cerebral cortex, explored with $\theta$ burst stimulation (TBS), was observed in $\mathrm{Cl}$ patients, and a positive correlation was found between amyloid- $\beta_{1-42}$ CSF contents and the magnitude of long-term potentiation-like effects induced by TBS. No correlation was conversely found between $\tau$ protein concentrations and MRI findings, cognitive parameters, and TBS effects in these patients. Together, our results indicate that in MS, central inflammation is able to alter amyloid- $\beta$ metabolism by reducing its concentration in the CSF and leading to impairment of synaptic plasticity and cognitive function.

Neuropsychopharmacology (20II) 36, 559-568; doi:I0.1038/npp.2010.187; published online I3 October 2010
\end{abstract}

Keywords: cognition; inflammation; LTP; $\tau$ protein; transcranial magnetic stimulation

\section{INTRODUCTION}

Multiple sclerosis (MS) causes cognitive deficits since the early stages of the disease, by altering memory, attention, and executive functions (Amato et al, 2004, 2006a, 2008a,b; Chiaravalloti and DeLuca, 2008; Genova et al, 2009).

The synaptic and molecular mechanisms at the basis of cognitive impairment in MS are still poorly understood. A large body of evidence converges in indicating that learning and memory processes are encoded in brain circuits as experience- and activity-dependent long-term synaptic changes. In particular, long-term potentiation (LTP) is a form of synaptic plasticity in which repeated stimulation connects neurons by making synapses more

\footnotetext{
*Correspondence: Dr D Centonze, Dipartimento di Neuroscienze, Clinica Neurologica, Università Tor Vergata, via Montpellier I, Rome 00133, Italy, Tel: + 3906725960 I0, Fax: + 390672596006 ,

E-mail: centonze@uniroma2.it

Received I4 May 2010; revised 9 September 2010; accepted II September 2010
}

responsive to future activation, and is commonly considered a major candidate for representing the substrate of learning and memory processes (Bliss and Collingridge, 1993; Malenka, 2003).

LTP induction is regulated by a variety of factors, able to interfere with critical receptor and post-receptor events at excitatory synapses (Bliss and Collingridge, 1993; Malenka, 2003; Feldman, 2009; Kessels and Malinow, 2009; Minichiello, 2009). Among these factors, amyloid- $\beta$, particularly the isoform amyloid- $\beta_{1-42}$, that was consistently found to be reduced in the cerebrospinal fluid (CSF) of Alzheimer's disease (AD) patients, has a major function in LTP deficits and cognitive impairment in $\mathrm{AD}$. More specifically, amyloid- $\beta_{1-42}$ aggregates in oligomers that impair synaptic plasticity mechanisms (Klyubin et al, 2005; Shankar et al, 2008; Walther et al, 2009; Townsend et al, 2010). Its role in other disorders associated with mental deterioration is, however, still unclear.

Amyloid- $\beta$ peptides are formed by proteolytic cleavage of a transmembrane protein, the amyloid precursor protein (APP) by $\beta$-site APP-cleaving enzyme 1 (BACE). BACE 
cleaves APP, causing the secretion of an extracellular soluble fragment of APP, termed sAPP- $\beta$, and the retention of a 99-residue $\mathrm{C}$-terminal fragment. This fragment can undergo further cleavage by $\gamma$-secretase to release 40 or 42 amino-acid long amyloid- $\beta$ fragments into the cytoplasm. However, APP may undergo at least two and most likely several different processing pathways. In the non-amyloidogenic pathway, APP is cleaved at the $\alpha$-site in the middle of the amyloid- $\beta$ domain. This cleavage obliterates amyloid$\beta$ formation, instead yielding an N-terminal sAPP- $\alpha$ fragment (Andreasson et al, 2007).

We postulated that, in MS, acute inflammation is able to interfere with amyloid- $\beta_{1-42}$ or $\tau$ metabolism, and that, like in $\mathrm{AD}$, this abnormal metabolism could be associated with cognitive dysfunction and synaptic plasticity. Notably, although both amyloid- $\beta_{1-42}$ and $\tau$ protein alter LTP induction and cognitive performances in animal models (Oddo et al, 2003; Klyubin et al, 2005; Rosenmann et al, 2008; Shankar et al, 2008; Polydoro et al, 2009; Walther et al, 2009; Townsend et al, 2010), their role in human synaptic plasticity has never been explored.

In humans, LTP can be explored in a safe and noninvasive manner by using paradigms of repetitive transcranial magnetic stimulation (rTMS) (Ziemann, 2004; Cooke and Bliss, 2006; Di Lazzaro et al, 2008). A novel LTPinducing protocol has been recently developed, in the attempt to mimic the physiological activity of hippocampal neurons during learning episodes (Huang et al, 2005; Di Lazzaro et al, 2008). This new protocol has been termed $\theta$ burst stimulation (TBS) and, according to in vitro experiments in hippocampal preparations, it produces an NMDA receptor-dependent LTP in the human cortex, by enhancing corticospinal excitability for several minutes after the end of the stimulation (Huang et al, 2005).

Thus, in this study, we investigated whether altered CSF concentrations of amyloid- $\beta_{1-42}$ metabolism are associated with abnormal patterns of cognitive performances and of cortical plasticity in a sample of MS patients that were submitted to an extensive neuropsychological examination and to neurophysiological investigations of cortical plasticity by means of TBS.

\section{SUBJECTS AND METHODS}

The study was approved by the Ethics Committee of the University Hospital Tor Vergata, Rome.

Abbreviations used throughout the text are provided in the Supplementary Text.

\section{MS Patients}

We collected CSF from 42 patients ( 30 females and 12 males, aged 23-54 years) admitted to the neurological clinic of the University Hospital Tor Vergata of Rome, and later diagnosed as suffering from clinically isolated syndrome (CIS) $(n=7)$, relapsing-remitting (RR) MS $(n=30)$, or primary progressive (PP) MS $(n=5)$. After their admittance, all patients underwent, in sequence, brain (and in selected cases also spinal) magnetic resonance imaging (MRI) scan, neuropsychological assessment, TMS, and CSF withdrawal within $24 \mathrm{~h}$. Corticosteroids or other MS-specific immunoactive therapies were initiated later when appropriate.
The diagnosis of CIS or MS was established by clinical, laboratory, and MRI parameters, and matched published criteria (McDonald et al, 2001; Polman et al, 2005). In all instances, patients underwent detection of oligoclonal banding in the CSF (positive in $92 \%$ of cases). Expanded disability status scale (EDSS) scores were between 0 and 5.

As controls, we used CSF from 12 age- and gendermatched individuals (eight females and four males, aged 25-52 years) without inflammatory or degenerative diseases of the central or peripheral nervous system. These subjects underwent lumbar puncture because of a clinical suspect of acute peripheral neuropathy, meningitis, or subarachnoidal hemorrhage, which were not confirmed. All the subjects gave their written informed consent to the study.

\section{MRI Acquisition and Analysis}

Three Tesla MRI scans consisted of dual-echo proton density, FLAIR, T2-weighted spin-echo images, and precontrast and post-contrast T1-weighted spin-echo images. All images were acquired in the axial orientation with $3 \mathrm{~mm}$-thick contiguous slices. T2 lesion volume was determined by manual tracing and the number of gadolinium-enhancing $(\mathrm{Gd}+)(0.2 \mathrm{ml} / \mathrm{kg}$ e.v. $)$ lesions was counted by a neuroradiologist who was unaware of the patient's clinical details (Centonze et al, 2007).

\section{CSF Determination of Amyloid- $\beta_{1-42}$ and Total $\tau$}

Immediately after its collection, CSF was centrifuged to eliminate cells and cellular debris and stored at $-80^{\circ} \mathrm{C}$ until analyzed (Centonze et al, 2007). Levels of both amyloid$\beta_{1-42}$ and total $\tau$ were determined according to standard procedures, using commercially available sandwich enzyme-linked immunosorbent assays (Innotest $\beta$-Amyloid 1-42, Innotest $\mathrm{h}-\tau \mathrm{Ag}$, Innogenetics, Ghent, Belgium) (Sancesario et al, 2010). Briefly, $25 \mu \mathrm{l}$ of the CSF from each patient were dispensed into corresponding 96-well ELISA plates, pre-coated either with the monoclonal antibody $21 \mathrm{~F} 12$ for amyloid- $\beta_{1-42}$, or AT120 for total $\tau$, and incubated, respectively, with the biotinylated antibody 3D6 or HT7. Bound antibodies were then detected by a peroxidase-labeled streptavidin, after addition of a substrate solution. The reaction was stopped by sulfuric acid. The absorbance of the reaction product was read at $450 \mathrm{~nm}$. The biomarker concentrations in the samples were calculated based on the amyloid- $\beta_{1-42}$ and $\tau$ standard sigmoid curve equation.

\section{Neuropsychological Assessment}

Cognitive functions were assessed through the Brief Repeatable Neuropsychological Battery (BRB) (Rao, 1990) by an expert trained clinician in a subgroup of $21 \mathrm{MS}$ patients (4 CIS and 17 RR MS) who gave consent to the examination. The BRB assesses the cognitive domains most frequently impaired in MS (Amato et al, 2004), and incorporates tests of verbal memory (selective reminding test (SRT)); visual memory (10/36 Spatial Recall Test); attention, concentration, and speed of information processing (paced auditory serial addition test (PASAT), symbol digit modalities test (SDMT)); and verbal fluency 
(word list generation). Performance on each test of the BRB was assessed by applying the available Italian normative values (Amato et al, 2006b). Failure of a test was defined when the score was at least 2 SD below the mean normative values.

Consistently with previous works (Amato et al, 2006a, 2008b), those patients who failed at least three tests were considered cognitively impaired (CI), and those who failed less than three tests were considered cognitively preserved (CP). No subjects were taking psychoactive drugs or substances that might interfere with neuropsychological performance.

\section{Transcranial Magnetic Stimulation}

TMS protocols were performed in a subgroup of $30 \mathrm{MS}$ patients (5 CIS, 23 RR MS, and 2 PP MS) who gave consent to the examination and were asymptomatic in the upper right limb. Electromyographic traces were recorded from the right first dorsal interosseus muscle (FDI) with surface cup electrodes. The active electrode was placed over the muscle belly and the reference electrode over the metacarpophalangeal joint of the index finger. Responses were amplified with a Digitimer D360 amplifier (Digitimer, Welwyn Garden City, Hertfordshire, UK) through filters set at $20 \mathrm{~Hz}$ and $2 \mathrm{kHz}$ with a sampling rate of $5 \mathrm{kHz}$, then recorded by a computer with SIGNAL software (Cambridge Electronic Devices, Cambridge, UK).

MEPs were evoked through a figure-of-eight coil with external loop diameter of $70 \mathrm{~mm}$ connected to a Magstim $200^{2}$ magnetic stimulator (Magstim Company, Whitland, Wales, UK). Coil position was adjusted to find the optimal scalp site to evoke motor responses in the contralateral FDI, the motor 'hot spot,' at the beginning of each experimental session and marked over the patient's scalp with a pencil. The coil was held tangentially to the scalp surface with the handle pointing posteriorly and laterally at about $45^{\circ}$ with respect to the mid-sagittal axis of the head.

\section{Intermittent TBS}

Intermittent TBS (iTBS) was delivered over the motor 'hot spot' of the right FDI through a Magstim Rapid ${ }^{2}$ stimulator. The active motor threshold (AMT) was defined as the minimum stimulation intensity required to evoke a liminal motor potential from the FDI during voluntary contraction (about $200 \mu \mathrm{V}$ in $50 \%$ of 10 trials). Stimulation intensity was $80 \%$ of AMT. The iTBS protocol consisted of 10 bursts, each burst composed of three stimuli at $50 \mathrm{~Hz}$, repeated at a $\theta$ frequency of $5 \mathrm{~Hz}$ every $10 \mathrm{~s}$ for a total of 600 stimuli (200 s). Sixty MEPs were collected before iTBS (baseline) and at two different time points ( 0 and $15 \mathrm{~min}$ ) after the end of iTBS. Stimulation intensity was set to induce a stable MEP of $\sim 1 \mathrm{mV}$ peak-to-peak amplitude in the relaxed right FDI at baseline and remained unchanged until end of recordings. MEP's amplitudes were then averaged at each time point and normalized to the mean baseline amplitude.

\section{Intracortical Circuits in Right M1}

We also tested, through paired-pulse (pp) TMS, shortinterval intracortical inhibition (SICI; mediated by intrinsic GABAAergic circuits) (Kujirai et al, 1993), intracortical facilitation (ICF; believed to follow the preferential recruitment of intrinsic excitatory fibers) (Kujirai et al, 1993), short intracortical facilitation (SICF; likely mediated by excitatory cortical interneurons) (Ziemann et al, 1998), and long-interval intracortical inhibition (LICI; mediated by local GABAB pathways) (Valls-Solé et al, 1992) of the left M1. One figure-of-eight coil, external diameter $70 \mathrm{~mm}$ was held tangentially to the scalp over the motor 'hot spot' for right FDI muscle. Stimulation intensity for TS was adjusted in each experiment to evoke an MEP of $\sim 1 \mathrm{mV}$ peakto-peak amplitude in the relaxed right FDI.

SICI and ICF were tested using ppTMS with a subthreshold CS preceding a suprathreshold TS (Kujirai et al, 1993; Rothwell, 1997). CS stimulus was set at $80 \%$ AMT. Three conditions were presented in a random order: control (TS given alone) and two pp conditions (TS preceded by CS) at one of two different ISI ( 2 and $10 \mathrm{~ms}$ ).

For SICF, the intensity of CS was set to $90 \%$ RMT. Six randomly intermixed conditions were presented in a random order: TS given alone and five conditions with the TS followed by CS at one of five different ISI $(1.5,2.1$, 2.7, 3.7, and $4.5 \mathrm{~ms}$ ) (Hanajima et al, 2002).

For LICI, the intensity of CS was set at $120 \%$ RMT. Two conditions were presented in a random order: control (TS given alone) and one paired-pulse condition (TS preceded by CS) at $100 \mathrm{~ms}$ ISI (Valls-Solé et al, 1992). For each experiment, 10 responses were collected for the test stimulus alone and for conditioned MEPs at each ISI. Changes in MEP amplitude at each ISI were expressed as the percentage of the mean unconditioned MEP amplitude.

\section{Data Analysis}

Differences in $\tau$ and amyloid- $\beta_{1-42}$ CSF levels were assessed using analysis of variance, followed by post hoc comparison using the Tukey highest significant difference procedure, to account for multiple comparisons and by $t$-test or Mann-Whitney test for comparisons between the two groups. Relationships between $\tau$ and amyloid- $\beta_{1-42}$ CSF levels and MRI findings were assessed using Spearman's correlation test for non-parametric values and Pearson's correlation test for parametric values. Correlation analysis between amyloid- $\beta_{1-42}$ CSF levels and cognitive variables was performed by calculating Spearman's correlation coefficients.

Correlations between $\tau$ and amyloid- $\beta_{1-42}$ CSF levels with iTBS at each time point, SICI, ICF, LICI, and SICF at each ISI were calculated using Pearson's correlation coefficient. Differences between groups were assessed using repeated measures analysis of variance for iTBS, SICF, SICI, ICF, and LICI. Bonferroni's correction was used to correct for multiple comparisons. Data, expressed as mean $\pm \mathrm{SEM}$, were considered significant at the 0.05 level.

\section{RESULTS}

CSF Contents of Amyloid- $\beta_{1-42}$ and total $\tau$ Protein in MS Patients and Their Correlation with Neuropsychological Parameters

We first measured amyloid- $\beta_{1-42}$ and $\tau$ protein levels in the CSF of MS patients. Amyloid- $\beta_{1-42}$ levels were significantly 
reduced in the population of MS subjects, compared with control individuals (MS group: $267 \pm 22 \mathrm{pg} / \mathrm{ml}, n=42$; control group: $390 \pm 45 \mathrm{pg} / \mathrm{ml}, n=12, p<0.05$ ) (Figure 1a). Conversely, $\tau$ protein levels were not altered in the CSF of MS patients (MS group: $128 \pm 11 \mathrm{pg} / \mathrm{ml}, n=42$; control group: $143 \pm 14 \mathrm{pg} / \mathrm{ml}, n=12$, NS) (Figure $1 \mathrm{~b}$ ).

MS patients were, then, classified as CI $(n=11)$ or CP $(n=10)$ on the basis of their neuropsychological performance, without differences in terms of demographic characteristics (CI: 7 females and 4 males, aged 25-40 years with $11 \pm 7$ years of education; CP: 6 females and 4 males, aged 23-37 years with $11 \pm 3$ years of education).

The reduction of amyloid- $\beta_{1-42}$ levels was related to cognitive dysfunction (CI MS group: $145 \pm 25 \mathrm{pg} / \mathrm{ml}, n=11$; CP MS group: $297 \pm 46 \mathrm{pg} / \mathrm{ml}, n=10$; control group: $390 \pm 45 \mathrm{pg} / \mathrm{ml}, n=12 ; \mathrm{F}=9.81, p=0.0005)$, as in CP MS amyloid- $\beta_{1-42}$ levels were higher than in CI MS patients $(p<0.05)$, and indistinguishable from controls (NS) (Figure 1c). In CI MS patients, $\tau$ protein CSF levels were conversely still indistinguishable from those measured in controls and in CP patients (CI MS group: $110 \pm 18 \mathrm{pg} / \mathrm{ml}$, CP MS group: $176 \pm 37 \mathrm{pg} / \mathrm{ml}$, control group: $143 \pm 14 \mathrm{pg} / \mathrm{ml}$, $\mathrm{F}=2.22, p=0.12)$ (Figure 1d).

The most frequently failed tests by examined MS patients were those assessing sustained attention and concentration (SDMT, PASAT) and verbal memory (SRT-LTS, SRTCLTR). Interestingly, a moderate positive correlation was found between amyloid- $\beta_{1-42}$ CSF levels and scores on measures of attention/concentration (SDMT: $n=21$, $r=0.51, \quad p=0.02 ; \quad$ PASAT: $n=21, \quad r=0.54, \quad p=0.01)$
(Figures 1e and f). The scores on verbal memory tests were, conversely, not significantly related to amyloid- $\beta$ contents (SRT-LTS: $n=21, r=-0.08, p=0.61$; SRT-CLTR: $n=21, r=-0.11, p=0.58)$. None of the neuropsychological measurements correlated to $\tau$ CSF levels in MS patients $(n=21, p=$ NS for all parameters, not shown).

As pathology of corpus callosum (CC) may have a major role in cognitive impairment of MS patients (Ozturk et al, 2010), we further compared scores in all neuropsychological tests between patients showing lesions within the CC $(n=6)$ and patients without CC lesions $(n=15)$ at conventional MRI. Comparison of neuropsychological scores between CIS and RRMS was also performed. No significant differences emerged between groups $(n=21$, all $p=\mathrm{NS})$ (not shown).

\section{Correlation Between Amyloid- $\beta_{1-42}$ and Total $\tau$ Protein with Gd + Lesions in MS Patients}

Upon grouping of MS patients according to the presence of $\mathrm{Gd}+$ lesions at the MRI (Gd + MS group: $n=22$ (14 patients complaining new symptoms since $\leqslant 10$ days), Gd-MS group: $n=20$ (four patients with new symptoms since $\leqslant 10$ days), control group: $n=12, \mathrm{~F}=7.98, p=0.0009$ ), amyloid$\beta_{1-42}$ levels were lower in $\mathrm{Gd}+$ MS patients $(209 \pm 26 \mathrm{pg} / \mathrm{ml})$ than in both Gd-MS patients $(331 \pm 25 \mathrm{pg} / \mathrm{ml}, p<0.05)$ and in controls $(390 \pm 45 \mathrm{pg} / \mathrm{ml}, p<0.01)$ (Figure 2a). No significant alteration of $\tau$ protein CSF content was revealed by grouping $\mathrm{Gd}-$ and $\mathrm{Gd}+$ patients $(\mathrm{Gd}+\mathrm{MS}$ group:
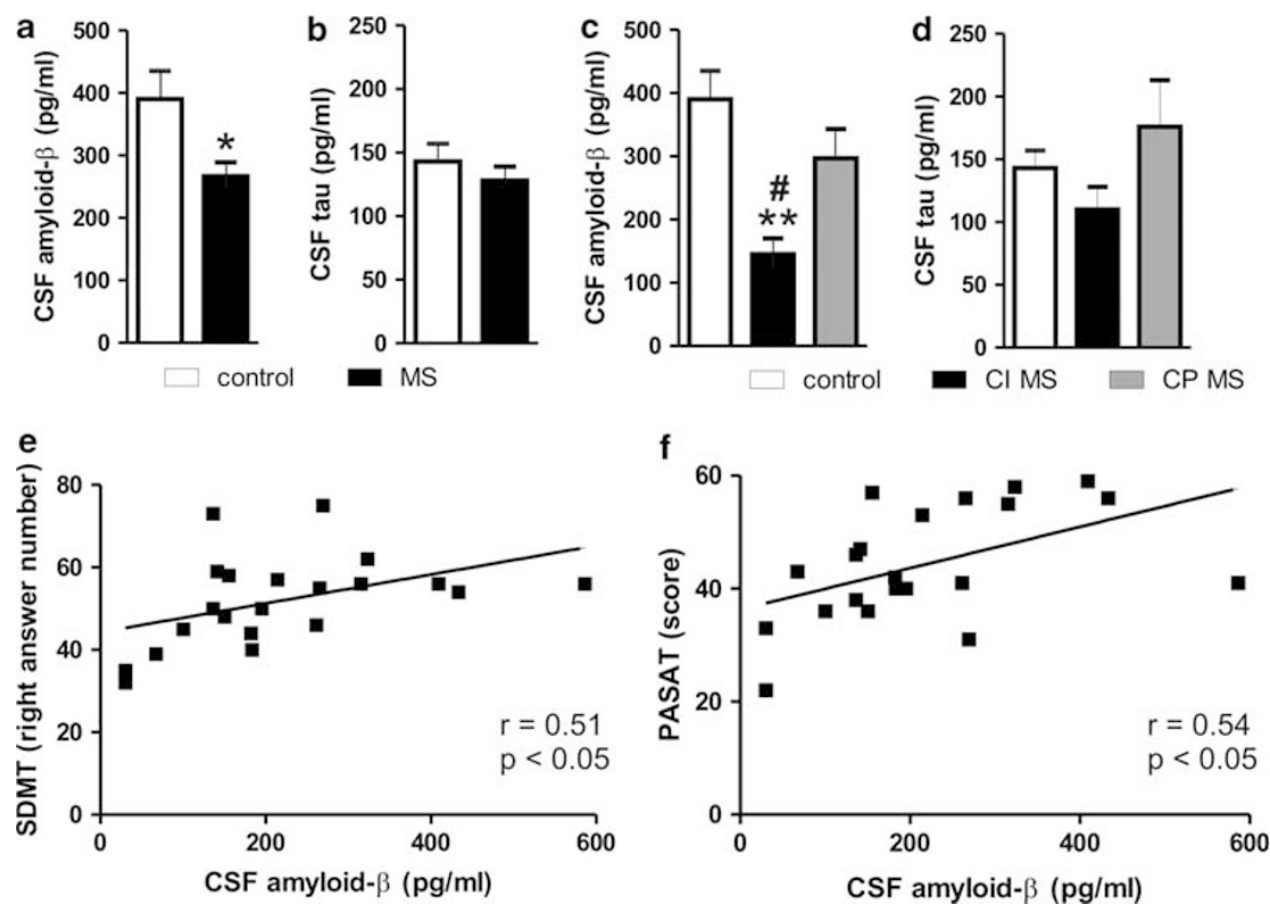

Figure I CSF contents of amyloid- $\beta_{1-42}$ are reduced in MS patients and correlate with their cognitive functions. (a) Amyloid- $\beta_{1-42}$ levels were significantly reduced in the CSF of MS subjects, compared with control individuals. (b) The $\tau$ protein levels were not altered in the CSF of MS patients. (c) In a subgroup of 2 I MS patients, who gave consent to the neuropsychological assessment, amyloid- $\beta_{1-42}$ levels were lower in cognitively impaired (Cl) $\mathrm{MS}$ patients than in both cognitively preserved (CP) MS patients and controls. (d) The $\tau$ protein CSF levels were normal also in CI MS patients. (e) Correlation plot between amyloid- $\beta_{1-42}$ CSF levels and PASAT score in MS subjects. (f) Correlation plot between amyloid- $\beta_{1-42}$ CSF levels and SDMT in MS subjects. In this figure and in the following ones, amyloid- $\beta_{1-42}$ is abbreviated in 'amyloid- $\beta$ ' $* p<0.05$ vs control $* * * 00.01$ vs control ${ }^{\#} p<0.05$ vs CP MS. PASAT, paced auditory serial addition test; SDMT, symbol digit modalities test. 

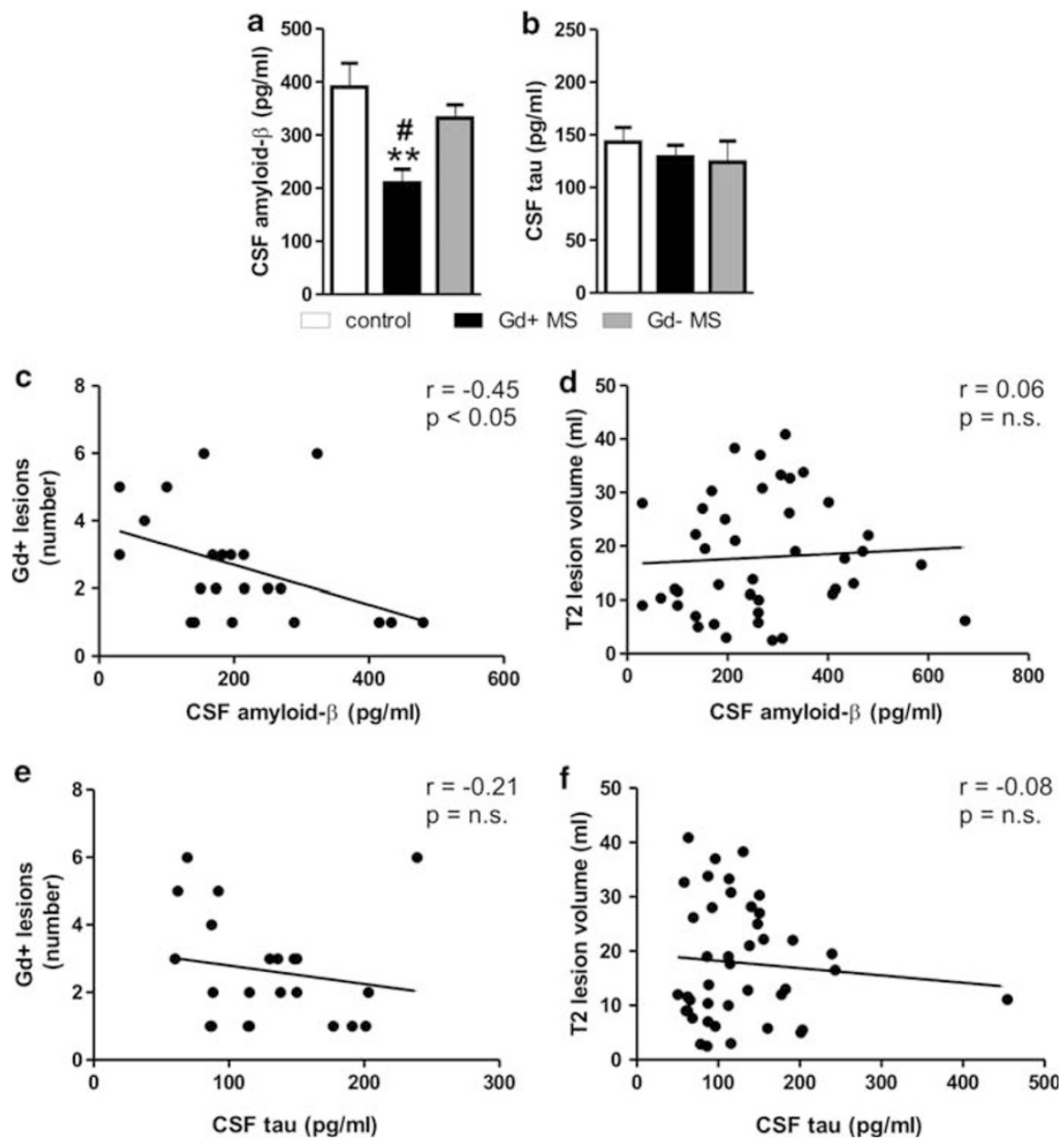

Figure 2 Disease activity at MRI correlates with reduction of amyloid- $\beta_{1-42}$ levels in the CSF of MS subjects. (a) The graph shows that, upon grouping of MS patients according to the presence of Gd + lesions, amyloid- $\beta_{1-42}$ levels were lower in Gd + MS subjects than in both Gd-MS subjects and controls. (b) The $\tau$ protein CSF levels were normal also in Gd + MS patients. (c) Correlation plot between amyloid- $\beta_{1-42}$ CSF levels and the number of Gd + lesions in Gd + MS subjects. (d) Correlation plot between amyloid- $\beta_{1-42}$ CSF levels and T2 lesion volume in MS subjects. (e, f) Correlation plots between $\tau$ CSF levels and the number of $\mathrm{Gd}+$ lesions (e) or T2 lesion volume in MS subjects ( $\mathrm{f}$. *** $p<0.0$ I vs control $\# p<0.05$ vs Gd-MS. Gd +, Gadolinium enhancing; Gd-, non-Gadolinium enhancing.

$129 \pm 11 \mathrm{pg} / \mathrm{ml}, \mathrm{Gd}-\mathrm{MS}$ group: $124 \pm 20 \mathrm{pg} / \mathrm{ml}$, control group: $143 \pm 14 \mathrm{pg} / \mathrm{ml} \mathrm{F}=0.3, p=0.74$ ) (Figure $2 \mathrm{~b}$ ).

These results might indicate that acute brain inflammation is associated with alterations of amyloid- $\beta$ metabolism, leading to decreased CSF detection of this biologically active peptide. We further explored this possibility by correlating both amyloid- $\beta_{1-42}$ and $\tau$ protein levels with the number of $\mathrm{Gd}+$ lesions seen at the MRI. These analyses uncovered a significant negative correlation between amyloid- $\beta_{1-42}$ content and $\mathrm{Gd}+$ lesions $(n=22, r=-0.45, p=0.03)$ (Figure 2c), whereas no correlation was seen between amyloid- $\beta_{1-42}$ and total lesion load in T2 MRI sequences $(n=42, r=0.06, p=0.69)$ (Figure 2d). No correlation was also found between $\tau$ levels in the CSF of MS patients and both Gd + lesions $(n=22, r=-0.21, p=0.34)$ and T2 lesion load ( $n=42, r=-0.08, p=0.57$ ) (Figures 2e and $\mathrm{f}$ ).

\section{Correlation Between TBS-Induced LTP with Amyloid- $\beta_{1-42}$ and Total $\tau$ Levels in MS Patients}

Amyloid- $\beta$ dimers isolated from AD brains completely block LTP induction in the rodent hippocampus, and consequently cause cognitive defects (Shankar et al, 2008). In an attempt to see if also in MS brains amyloid- $\beta_{1-42}$ is able to alter LTP induction, we explored whether any correlation could be uncovered between CSF content of amyloid- $\beta_{1-42}$ and the magnitude of LTP induced in MS patients with TBS. A significant positive correlation was found between the magnitude of LTP measured $15 \mathrm{~min}$ after the TBS protocol and the levels of amyloid- $\beta_{1-42}$ in the CSF $(r=0.48, p=0.007)$, a finding that may suggest that also in $\mathrm{MS}$, as in $\mathrm{AD}$, reduced CSF contents of amyloid- $\beta_{1-42}$ may be associated with defective memory-related synaptic plasticity (Figures $3 a$ and $b$ ).

\section{Correlation Between Neuropsychological Assessments and TBS-Induced LTP}

To investigate whether cognitive impairment was associated with reduced cortical LTP in MS subjects, we compared the mean percentage values of peak-to-peak MEP amplitudes before and after TBS in CI and CP MS patients. A repeatedmeasures ANOVA with TIME (3 levels: baseline, 0, and 15 min post-TBS) as within subjects and GROUP (2 levels: 

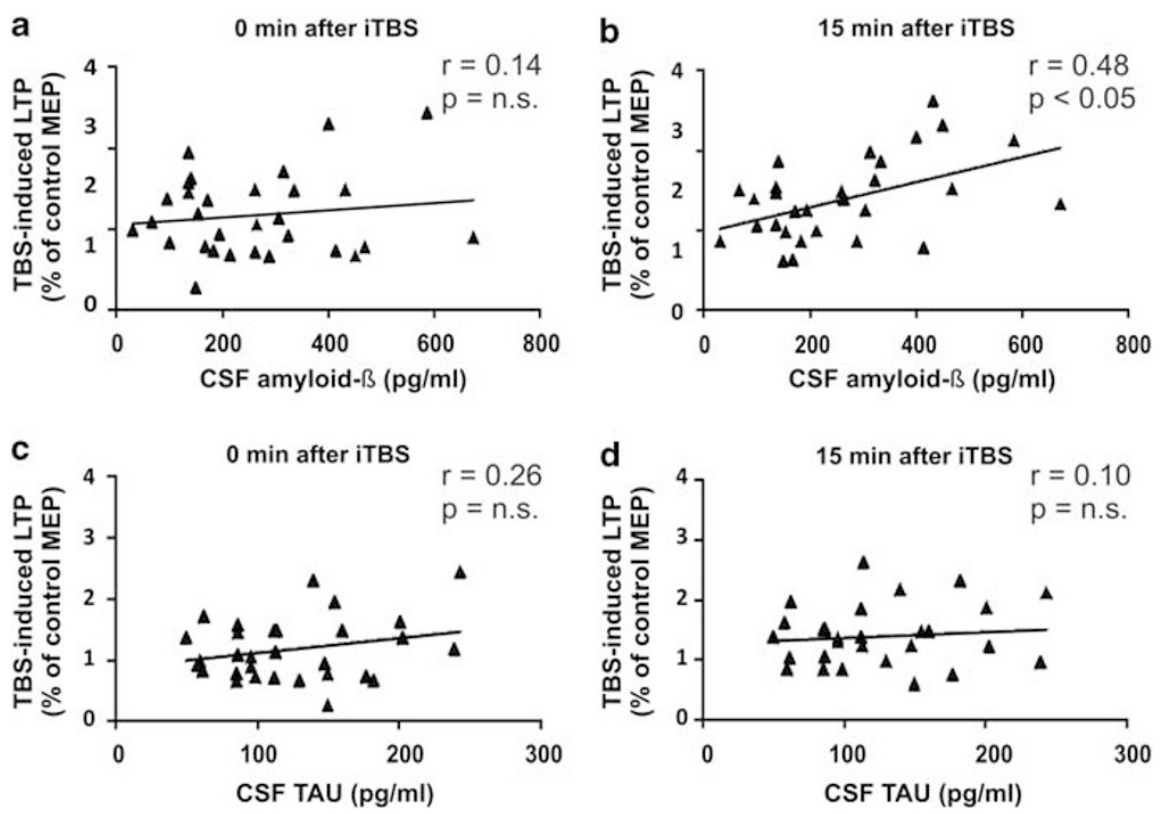

Figure 3 TBS-induced LTP magnitude correlates with amyloid- $\beta_{1-42}$ levels in the CSF of MS subjects. (a) Correlation plot between amyloid- $\beta_{1-42}$ CSF levels and MEP amplitude changes, expressed as percentage of baseline, immediately after TBS, and (b) I 5 min after TBS. (c, d) Correlation plots between $\tau$ CSF levels and TBS-induced LTP. LTP, long-term potentiation; TBS, $\theta$ burst stimulation.

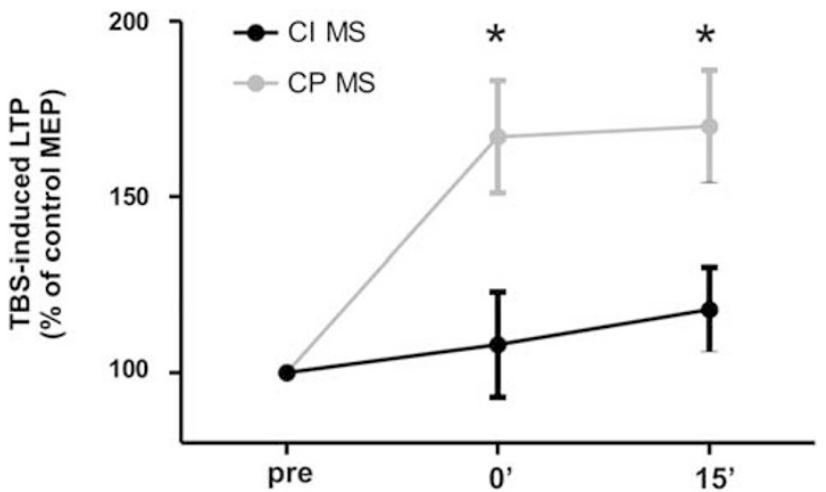

Figure 4 TBS-induced LTP differs between cognitively impaired and cognitively preserved MS patients. Differences between the two groups were observed at both 0 and 15 min after TBS time points. $* p<0.05$.

$\mathrm{CI}$ and $\mathrm{CP}$ ) as between subjects main factors showed a significant effect of time $(\mathrm{F}=8.04, p=0.002)$, a significant effect of GROUP $(\mathrm{F}=6.82, p=0.023)$ and a significant TIME $\times$ GROUP interaction $(\mathrm{F}=5.96, p=0.031)$. At post hoc contrasts CI patients showed a lower effect of TBS at both time points $(0 \mathrm{~min}$ post-TBS: $1.09 \pm 0.14 ; 15 \mathrm{~min}$ post: $1.18 \pm 0.12$; all $p<0.05)$ compared with $\mathrm{CP}$ patients $(0 \mathrm{~min}$ post-TBS: $1.67 \pm 0.16 ; 15 \mathrm{~min}$ post: $1.71 \pm 0.16$ ) (Figure 4 ). We also performed a $t$-test for independent data on the peak effect of TBS represented by the post-TBS time point with the highest mean MEP amplitude. A significantly lower peak effect of TBS in CI $(n=10, \quad$ mean $\pm \mathrm{SE}=1.39 \pm 0.15)$ compared with $\mathrm{CP}(n=10$, mean $\pm \mathrm{SE}=2.32 \pm 0.23)$ MS patients $(p=0.008)$ was observed (not shown). These findings, showing a lower effect of TBS in CI patients, are in line with previous observations that rTMS has an altered effect in AD patients (Inghilleri et al, 2006; Battaglia et al, 2007) and show for the first time that cognitive impairment in MS patients is paralleled by an alteration of NMDARdependent synaptic plasticity. Comparison of TBS effect between CIS, RRMS, and PPMS did not show any significant difference.

Role of Amyloid- $\beta_{1-42}$ in the Modulation of Excitatory and Inhibitory Synaptic Transmission in MS

LTP is a complex phenomenon, and changes in glutamatemediated synaptic drive or GABA-mediated inhibition alter its induction. Accordingly, blockade of NMDA glutamate receptors prevents LTP (Bliss and Collingridge, 1993; Cooke and Bliss, 2006), as also does the enhancement of GABAmediated inhibition (Matsuyama et al, 2008; Gong et al, 2009; Pan et al, 2009). Thus, we tried to investigate whether amyloid- $\beta_{1-42}$-mediated inhibition of LTP could be secondary to impaired basal synaptic transmission in cortical neurons of MS patients. In ppTMS experiments, however, we found that the degree of SICI, ICF, SICF, and LICI was not related to the CSF levels of amyloid- $\beta_{1-42}$ in MS patients, suggesting that this protein affects synaptic plasticity but not basal excitatory or inhibitory transmission (Figure 5). Comparisons between CI and CP MS patients also did not reveal any significant difference (not shown).

\section{DISCUSSION}

Gray matter pathology, brain atrophy, and loss of anatomical connectivity are the most accredited determinants of progressive cognitive decline in MS (Calabrese et al, 2009, Calabrese et al, 2010; Staff et al, 2009; Tiemann et al, 2009). Acute and reversible mental deterioration also occurs during clinical relapses, independently of the primary functional system involved (Foong et al, 1998). The presence of $\mathrm{Gd}+$ lesions at the brain MRI also 

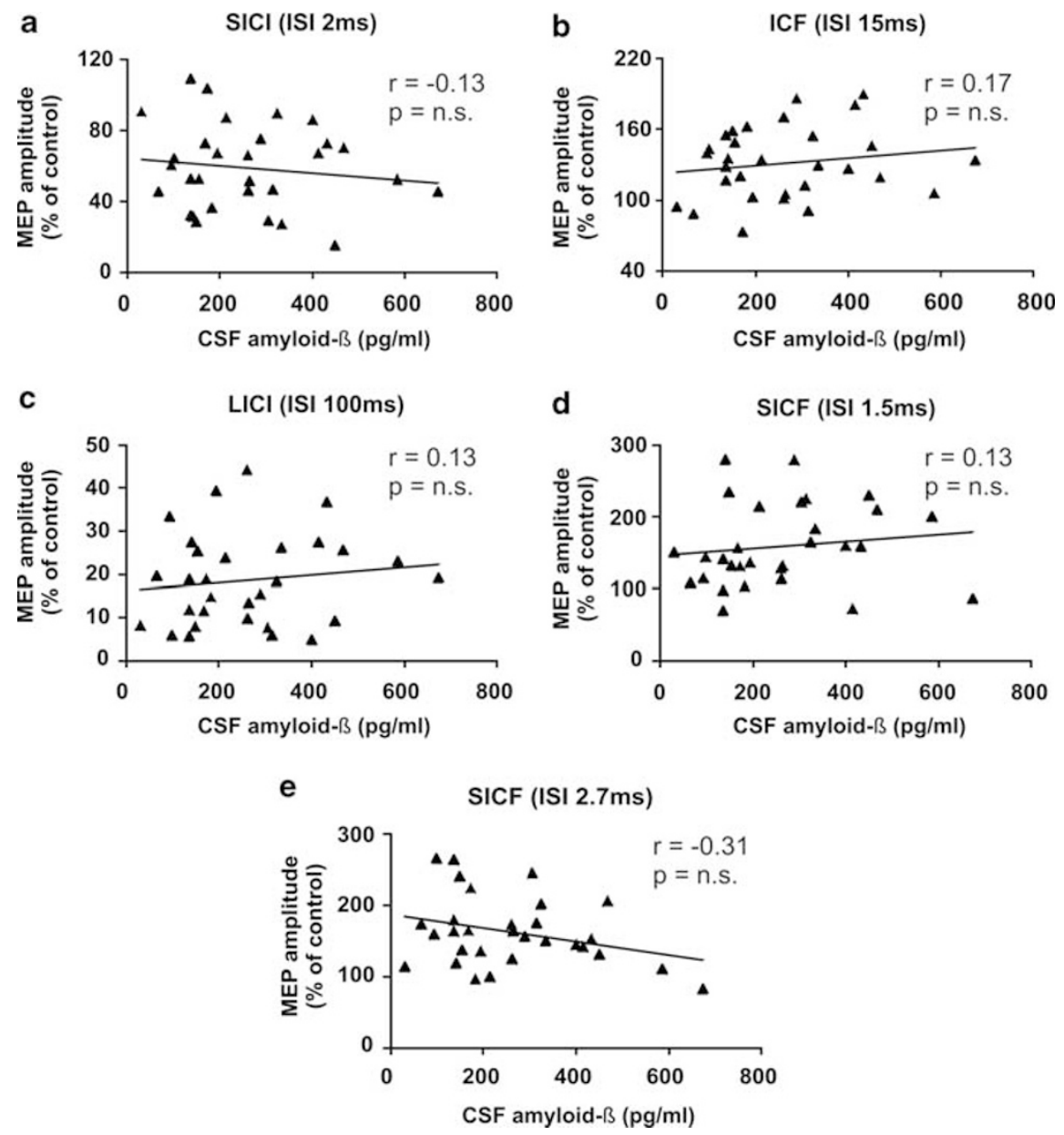

Figure 5 Correlation plots between amyloid- $\beta_{1-42}$ CSF levels and MEP amplitude changes, expressed as percentage of baseline, induced by (a) SICl at $2 \mathrm{~ms} \mathrm{ISI,} \mathrm{(b)} \mathrm{ICF} \mathrm{at} \mathrm{I} 0 \mathrm{~ms} \mathrm{ISI,} \mathrm{(c)} \mathrm{LICl} \mathrm{at} 100 \mathrm{~ms} I S I$, (d) SICF at I.5 ms ISI, and (e) at $2.7 \mathrm{~ms} I S I$. MEP, motor-evoked potential; SICl, short-interval intracortical inhibition; ISI, interstimulus interval; ICF, intracortical facilitation; LICI, long-interval intracortical inhibition; SICF, short intracortical facilitation.

correlates with poor cognitive performance in asymptomatic and symptomatic MS patients (Foong et al, 1998; Bellmann-Strobl et al, 2009), suggesting that focal inflammation is able to diffusely disrupt the neuronal substrate of information processing, leading to acute cognitive impairment. Given that amyloid- $\beta_{1-42}$ metabolism is regulated by inflammation (Griffin et al, 2006; Hickman et al, 2008; Schmidt et al, 2008), a recent study performed in a large sample of subjects reported that in MS patients there is an overall decrease of CSF amyloid- $\beta_{1-42}$ in comparison with healthy controls (Mattsson et al, 2009). These results seem to indicate that in MS there could be an altered expression of amyloid- $\beta_{1-42}$, possibly related to the underlying inflammatory processes.

This study shows that during the inflammatory phase of MS, amyloid- $\beta_{1-42}$ metabolism is altered leading to its reduced detection in the CSF. We found, in fact, that amyloid- $\beta_{1-42}$ levels were significantly lower in subjects with Gd + lesions at the MRI. Interestingly, these findings are in good agreement with previous studies showing that IL-1 $\beta$, an important pro-inflammatory cytokine, alters amyloid- $\beta$ metabolism in the cerebral cortex, increasing APP levels in rat brain homogenate (Fan et al, 2009). There is no information, conversely, about IL- $1 \beta$ effect on CSF amyloid- $\beta_{1-42}$ levels. However, inflammation increases
BACE activity (Hong et al, 2003), and BACE activity levels were found to be higher in patients with low amyloid $\beta_{1-42}$ levels in the CSF (Mulder et al, 2010). Anti-inflammatory cytokines, on the other hand, enhance the activity of amyloid- $\beta$ degrading enzymes, thus favoring amyloid- $\beta$ tissue deposit clearance (Shimizu et al, 2008).

This study shows, for the first time, a difference in cognitive profile of MS patients related to CSF levels of amyloid- $\beta_{1-42}$. Interestingly, the cognitive domains that seemed to be affected in association with low amyloid- $\beta$ CSF levels were those regarding attention, concentration, and information-processing speed, the same influenced by the presence of radiological disease activity (Bellmann-Strobl et al, 2009). Gd enhancement has been associated with poor PASAT performance in otherwise physically stable MS patients (Bellmann-Strobl et al, 2009) and to CSF amyloid$\beta_{1-42}$ levels (this work), in line with the idea that CSF amyloid- $\beta_{1-42}$ expression, altered by brain inflammation, may cause a diffuse impairment of cerebral connectivity with a negative impact on cognitive functioning. Conversely, neuropsychological test scores examining memory did not correlate with amyloid- $\beta_{1-42}$ levels.

Cortical atrophy was not measured in this study as imaging was performed for clinical purposes, thus only conventional MRI scans were collected. However, CSF $\tau$ 
levels, already reported to be associated with neurodegenerative damage (Vemuri et al, 2009; Ghoshal et al, 2002) were found to be normal in our patients. A further limitation of this study may be represented by anxiety, because of diagnosis communication or expectancy for the disease. Anxiety is known to influence the performance in the neuropsychological tests (Peretti, 1998; Tombaugh, 2006), especially those addressing concentration and attention. However, all participants were diagnosed as suffering from CIS or MS during hospitalization, and diagnosis was communicated after all data collection.

The synaptotoxic properties of amyloid- $\beta_{1-42}$ have long been recognized, as well as its ability to interfere with LTP and with cognitive abilities (Klyubin et al, 2005; Battaglia et al, 2007; Shankar et al, 2008; Nygaard and Strittmatter, 2009; Walther et al, 2009; Townsend et al, 2010). A recent study reported that amyloid- $\beta$ dimers isolated from $\mathrm{AD}$ patients can impair hippocampal LTP and memory in mice and induce dendritic spine retraction in neurons (Shankar et al, 2008). Of note, although the role of amyloid- $\beta$ in LTP disruption has been shown in a variety of experimental models of AD (Klyubin et al, 2005; Battaglia et al, 2007; Shankar et al, 2008; Walther et al, 2009; Townsend et al, 2010), its correlation with LTP measured in humans have never been shown before. Thus, the correlation between amyloid- $\beta_{1-42}$ CSF levels and LTP amplitude reported in this study is a first indication that amyloid- $\beta$ is a potent regulator of synaptic plasticity also in the human brain, with potentially relevant consequences for understanding the mechanisms of cognitive deficits not only in MS subjects but also in other neurological disorders, obviously including $\mathrm{AD}$. Importantly, although both altered cognitive performances and LTP induction was found in mice overexpressing $\tau$ protein (Polydoro et al, 2009), $\tau$ levels did not correlate with TBS-induced LTP amplitude in MS patients (Figures $3 \mathrm{c}$ and $\mathrm{d}$ ). In this respect, it is noteworthy that only LTP induced by high-frequency stimulation but not that induced by TBS was altered in mice overexpressing $\tau$ (Polydoro et al, 2009).

Previous investigations found that in MS patients SICI was lower in secondary progressive MS (SPMS) in comparison with RRMS patients in the remitting phase, and that SICI correlated significantly with EDSS scores (Conte et al, 2009). These results are in apparent contrast with our findings. However, our study group did not include patients with SPMS, and the mean age and disease duration of these patients (age: $51 \pm 0$, disease duration: $21.0 \pm 2$ ) are also very different in comparison with our study group (age: $34.3 \pm 1.4$ years, disease duration: $2.7 \pm 0.8$ years). Other investigators found that fatigue in MS is associated with lower SICI compared with MS patients without fatigue and healthy subjects (Liepert et al, 2005). In our study group, only very few patients complained fatigue and also in this case our group differed in terms of mean age (41.4 \pm 4.75 years). Moreover, one of the inclusion criteria in the study from Liepert was that patients had to be in the remitting phase of the disease, thus presumably also had longer disease durations. Finally, a similar reduction of SICI was found in the relapsing phase of the disease in comparison with remitting patients in a study by Caramia et al (2004). Moreover, in this case, the study group had longer disease duration (mean $4.29 \pm 3.26$ years), included only patients with a definite diagnosis of RRMS. Thus, differences in the results may be explained by the different clinical characteristics of the samples studied.

A recent investigation based on a different TMS protocol to induce neuroplastic changes, suggested that motor plasticity in MS patients is comparable with that found in healthy subjects (Zeller et al, 2010). Neuroplasticity in this study was, however, assessed only in patients in the remitting phase of the disease, with a stable clinical condition, whereas the alterations observed in our study were evident in patients with cognitive impairment and the presence of Gd + lesions.

The reduction in CSF amyloid- $\beta_{1-42}$ in $\mathrm{AD}$ has been postulated to reflect deposition of the aggregated insoluble fibrillar amyloid- $\beta$ peptides in senile plaque, with lower levels of diffusion into the CSF. A limitation of this study is that we do not provide any evidence of amyloid- $\beta$ tissue deposition in our patients. We cannot argue, therefore, that the decrease in amyloid- $\beta_{1-42}$ CSF levels observed is consequent to tissue deposition. However, AD neuropathology apart from amyloid- $\beta$ deposition includes variable amounts of soluble species that show much stronger correlations with severity of dementia than fibrillar amyloid- $\beta$ (Terry, 1996; Lue et al, 1999; McLean et al, 1999; Gandy et al, 2010). Synthetic amyloid- $\beta$ dimers are extremely potent inhibitors of synaptic plasticity both in vivo and in vitro, whereas even relatively high concentrations of amyloid- $\beta$ monomers are inactive (Selkoe, 2008; $\mathrm{Hu}$ et al, 2008; Shankar et al, 2008). Moreover, human ex vivo samples of CSF that contain amyloid- $\beta$ oligomers but not monomers potently inhibit LTP (Klyubin et al, 2005). The lowering of natively measured amyloid- $\beta_{1-42}$ in the CSF of our patients may be consequent to oligomerization as observed in AD (Englund et al, 2009). Finally, a transgenic mice overexpressing $\mathrm{APP}^{\mathrm{E} 693 \mathrm{Q}}$ develops learning and memory deficits in association with the formation of amyloid- $\beta$ oligomers without generating amyloid plaques in their brains at any age (Gandy et al, 2010). These observations, even though only at a speculative stage, could help explaining the findings of our iTBS experiments. Another possibility is that the observed findings could be related to inflammation-induced downregulation of $\beta$-site APP cleavage. Accordingly, BACE activity is modulated by inflammation (Mattsson et al, 2009) and is also involved in the promotion of myelination by oligodendrocytes (Hu et al, 2006).

Nevertheless, the mechanism by which amyloid- $\beta_{1-42}$ is reduced during inflammation in MS remains unclear, and further studies are needed to better clarify this issue.

\section{ACKNOWLEDGEMENTS}

This investigation was supported by the Italian National Ministero dell'Università e della Ricerca to DC, by the Italian National Ministero della Salute, and by Fondazione TERCAS to DC.

\section{DISCLOSURE}

The authors declare no conflict of interest. 


\section{REFERENCES}

Amato MP, Bartolozzi ML, Zipoli V, Portaccio E, Mortilla M, Guidi L et al (2004). Neocortical volume decrease in relapsing-remitting MS patients with mild cognitive impairment. Neurology 63: 89-93.

Amato MP, Zipoli V, Goretti B, Portaccio E, De Caro MF, Ricchiuti L et al (2006a). Benign multiple sclerosis: cognitive, psychological and social aspects in a clinical cohort. J Neurol 253: 1054-1059.

Amato MP, Portaccio E, Goretti B, Zipoli V, Ricchiuti L, De Caro MF et al (2006b). The Rao's brief repeatable battery and stroop test: normative values with age, education and gender corrections in an Italian population. Mult Scler 12: 787-793.

Amato MP, Zipoli V, Portaccio E (2008a). Cognitive changes in multiple sclerosis. Expert Rev Neurother 8: 1585-1596.

Amato MP, Portaccio E, Stromillo ML, Goretti B, Zipoli V, Siracusa $\mathrm{G}$ et al (2008b). Cognitive assessment and quantitative magnetic resonance metrics can help to identify benign multiple sclerosis. Neurology 71: 632-638.

Andreasson U, Portelius E, Andersson ME, Blennow K, Zetterberg $\mathrm{H}$ Aspects of beta-amyloid as a biomarker for Alzheimer's disease. Biomark Med, 2007 1: 59-78.

Battaglia F, Wang HY, Ghilardi MF, Gashi E, Quartarone A, Friedman E et al (2007). Cortical plasticity in Alzheimer's disease in humans and rodents. Biol Psychiatry 62: 1405-1412.

Bellmann-Strobl J, Wuerfel J, Aktas O, Dörr J, Wernecke KD, Zipp F et al (2009). Poor PASAT performance correlates with MRI contrast enhancement in multiple sclerosis. Neurology 73: 1624-1627.

Bliss TV, Collingridge GL (1993). A synaptic model of memory: long-term potentiation in the hippocampus. Nature 361: 31-39.

Calabrese M, Agosta F, Rinaldi F, Mattisi I, Grossi P, Favaretto A et al (2009). Cortical lesions and atrophy associated with cognitive impairment in relapsing-remitting multiple sclerosis. Arch Neurol 66: 1144-1150.

Calabrese M, Rinaldi F, Mattisi I, Grossi P, Favaretto A, Atzori $\mathrm{M}$ et al (2010). Widespread cortical thinning characterizes patients with MS with mild cognitive impairment. Neurology 74: 318-321.

Caramia MD, Palmieri MG, Desiato MT, Boffa L, Galizia P, Rossini PM et al (2004). Brain excitability changes in the relapsing and remitting phases of multiple sclerosis: a study with transcranial magnetic stimulation. Clin Neurophysiol 115: 956-965.

Centonze D, Bari M, Rossi S, Prosperetti C, Furlan R, Fezza F et al (2007). The endocannabinoid system is dysregulated in multiple sclerosis and in experimental autoimmune encephalomyelitis. Brain 130: 2543-2553.

Chiaravalloti ND, DeLuca J (2008). Cognitive impairment in multiple sclerosis. Lancet Neurol 7: 1139-1151.

Conte A, Lenzi D, Frasca V, Gilio F, Giacomelli E, Gabriele M et al (2009). Intracortical excitability in patients with relapsingremitting and secondary progressive multiple sclerosis. J Neurol 256: 933-938.

Cooke SF, Bliss TV (2006). Plasticity in the human central nervous system. Brain 129: 1659-1673.

Di Lazzaro V, Pilato F, Dileone M, Profice P, Oliviero A, Mazzone P et al (2008). The physiological basis of the effects of intermittent theta burst stimulation of the human motor cortex. J Physiol 586: 3871-3879.

Englund H, Degerman Gunnarsson M, Brundin RM, Hedlund M, Kilander L, Lannfelt L et al (2009). Oligomerization partially explains the lowering of Abeta42 in Alzheimer's disease cerebrospinal fluid. Neurodegener Dis 6: 139-147.

Fan LW, Mitchell HJ, Tien LT, Rhodes PG, Cai Z (2009). Interleukin-1beta-induced brain injury in the neonatal rat can be ameliorated by alpha-phenyl-n-tert-butyl-nitrone. Exp Neurol 220: $143-153$.
Feldman DE (2009). Synaptic mechanisms for plasticity in neocortex. Annu Rev Neurosci 32: 33-55.

Foong J, Rozewicz L, Quaghebeur G, Thompson AJ, Miller DH, Ron MA (1998). Neuropsychological deficits in multiple sclerosis after acute relapse. J Neurol Neurosurg Psychiatry 64: 529-532.

Gandy S, Simon AJ, Steele JW, Lublin AL, Lah JJ, Walker LC et al (2010). Days to criterion as an indicator of toxicity associated with human Alzheimer amyloid-beta oligomers. Ann Neurol 68: 220-230.

Genova HM, Sumowski JF, Chiaravalloti N, Voelbel GT, Deluca J (2009). Cognition in multiple sclerosis: a review of neuropsychological and fMRI research. Front Biosci 14: 1730-1744.

Ghoshal N, García-Sierra F, Wuu J, Leurgans S, Bennett DA, Berry RW et al (2002). Tau conformational changes correspond to impairments of episodic memory in mild cognitive impairment and Alzheimer's disease. Exp Neurol 177: 475-493.

Gong N, Li Y, Cai GQ, Niu RF, Fang Q, Wu K et al (2009). GABA transporter-1 activity modulates hippocampal theta oscillation and theta burst stimulation-induced long-term potentiation. J Neurosci 29: 15836-15845.

Griffin WS, Liu L, Li Y, Mrak RE, Barger SW (2006). Interleukin-1 mediates Alzheimer and Lewy body pathologies. J Neuroinflammation 3: 5.

Hanajima R, Ugawa Y, Terao Y, Enomoto H, Shiio Y, Mochizuki H et al (2002). Mechanisms of intracortical I-wave facilitation elicited with paired-pulse magnetic stimulation in humans. J Physiol 538: 253-261.

Hickman SE, Allison EK, El Khoury J (2008). Microglial dysfunction and defective beta-amyloid clearance pathways in aging Alzheimer's disease mice. J Neurosci 28: 8354-8360.

Hong HS, Hwang EM, Sim HJ, Cho HJ, Boo JH, Oh SS et al (2003). Interferon gamma stimulates beta-secretase expression and sAPPbeta production in astrocytes. Biochem Biophys Res Commun 307: 922-927.

Hu NW, Smith IM, Walsh DM, Rowan MJ (2008). Soluble amyloidbeta peptides potently disrupt hippocampal synaptic plasticity in the absence of cerebrovascular dysfunction in vivo. Brain 131: 2414-2424.

Hu X, Hicks CW, He W, Wong P, Macklin WB, Trapp BD et al (2006). Bacel modulates myelination in the central and peripheral nervous system. Nat Neurosci 9: 1520-1525.

Huang YZ, Edwards MJ, Rounis E, Bhatia KP, Rothwell JC (2005). Theta burst stimulation of the human motor cortex. Neuron 45: 201-206.

Inghilleri M, Conte A, Frasca V, Scaldaferri N, Gilio F, Santini M et al (2006). Altered response to rTMS in patients with Alzheimer's disease. Clin Neurophysiol 117: 103-109.

Kessels HW, Malinow R (2009). Synaptic AMPA receptor plasticity and behavior. Neuron 61: 340-350.

Klyubin I, Walsh DM, Lemere CA, Cullen WK, Shankar GM, Betts $\mathrm{V}$ et al (2005). Amyloid beta protein immunotherapy neutralizes Abeta oligomers that disrupt synaptic plasticity in vivo. Nat Med 11: 556-561.

Kujirai T, Caramia MD, Rothwell JC, Day BL, Thompson PD, Ferbert A et al (1993). Corticocortical inhibition in human motor cortex. J Physiol 471: 501-519.

Liepert J, Mingers D, Heesen C, Bäumer T, Weiller C (2005). Motor cortex excitability and fatigue in multiple sclerosis: a transcranial magnetic stimulation study. Mult Scler 11: 316-321.

Lue LF, Kuo YM, Roher AE, Brachova L, Shen Y, Sue L et al (1999). Soluble amyloid beta peptide concentration as a predictor of synaptic change in Alzheimer's disease. Am J Pathol 155: 853-862.

Malenka RC (2003). The long-term potential of LTP. Nat Rev Neurosci 4: 923-926.

Matsuyama S, Taniguchi T, Kadoyama K, Matsumoto A (2008). Long-term potentiation-like facilitation through GABAA receptor blockade in the mouse dentate gyrus in vivo. Neuroreport 19: 1809-1813. 
Mattsson N, Axelsson M, Haghighi S, Malmeström C, Wu G, Anckarsäter R et al (2009). Reduced cerebrospinal fluid BACE1 activity in multiple sclerosis. Mult Scler 15: 448-454.

McDonald WI, Compston A, Edan G, Goodkin D, Hartung HP, Lublin FD et al (2001). Recommended diagnostic criteria for multiple sclerosis: guidelines from the International Panel on the diagnosis of multiple sclerosis. Ann Neurol 50: 121-127.

McLean CA, Cherny RA, Fraser FW, Fuller SJ, Smith MJ, Beyreuther K et al (1999). Soluble pool of A beta amyloid as a determinant of severity of neurodegeneration in Alzheimer's disease. Ann Neurol 46: 860-866.

Minichiello L (2009). TrkB signalling pathways in LTP and learning. Nat Rev Neurosci 10: 850-860.

Mulder SD, van der Flier WM, Verheijen JH, Mulder C, Scheltens P, Blankenstein MA et al (2010). BACE1 activity in cerebrospinal fluid and its relation to markers of AD pathology. J Alzheimers Dis 20: 253-260.

Nygaard HB, Strittmatter SM (2009). Cellular prion protein mediates the toxicity of beta-amyloid oligomers: implications for Alzheimer disease. Arch Neurol 66: 1325-1328.

Oddo S, Caccamo A, Shepherd JD, Murphy MP, Golde TE, Kayed R et al (2003). Triple-transgenic model of Alzheimer's disease with plaques and tangles: intracellular Abeta and synaptic dysfunction. Neuron 39: 409-421.

Ozturk A, Smith SA, Gordon-Lipkin EM, Harrison DM, Shiee N, Pham DL et al (2010). MRI of the corpus callosum in multiple sclerosis: association with disability. Mult Scler 16: 166-177.

Pan BX, Dong Y, Ito W, Yanagawa Y, Shigemoto R, Morozov A (2009). Selective gating of glutamatergic inputs to excitatory neurons of amygdala by presynaptic GABAb receptor. Neuron 61: 917-929.

Peretti CS (1998). Anxiety and cognition disorders. Encephale 24: 256-259.

Polman CH, Reingold SC, Edan G, Filippi M, Hartung HP, Kappos L et al (2005). Diagnostic criteria for multiple sclerosis: 2005 revisions to the 'McDonald Criteria'. Ann Neurol 58: 840-846.

Polydoro M, Acker CM, Duff K, Castillo PE, Davies P (2009). Agedependent impairment of cognitive and synaptic function in the htau mouse model of tau pathology. J Neurosci 29: 10741-10749.

Rao S (1990). A Manual for the Brief Repeatable Battery of Neuropsychological Tests in Multiple Sclerosis. Medical College of Wisconsin: Milwaukee.

Rosenmann H, Grigoriadis N, Eldar-Levy H, Avital A, Rozenstein $\mathrm{L}$, Touloumi $\mathrm{O}$ et al (2008). A novel transgenic mouse expressing double mutant tau driven by its natural promoter exhibits tauopathy characteristics. Exp Neurol 212: 71-84.

Rothwell JC (1997). Techniques and mechanisms of action of transcranial stimulation of the human motor cortex. J Neurosci Methods 74: 113-122.

Sancesario GM, Esposito Z, Nuccetelli M, Bernardini S, Sorge R, Martorana A et al (2010). Abeta1-42 detection in CSF of Alzheimer's disease is influenced by temperature: indication of reversible Abeta1-42 aggregation? Exp Neurol 223: 371-376.
Selkoe DJ (2008). Soluble oligomers of the amyloid beta-protein impair synaptic plasticity and behavior. Behav Brain Res 192: 106-113.

Shankar GM, Li S, Mehta TH, Garcia-Munoz A, Shepardson NE, Smith I et al (2008). Amyloid-beta protein dimers isolated directly from Alzheimer's brains impair synaptic plasticity and memory. Nat Med 14: 837-842.

Schmidt J, Barthel K, Wrede A, Salajegheh M, Bähr M, Dalakas MC (2008). Interrelation of inflammation and APP in sIBM: IL-1 beta induces accumulation of beta-amyloid in skeletal muscle. Brain 131: $1228-1240$.

Shimizu E, Kawahara K, Kajizono M, Sawada M, Nakayama H (2008). IL-4-induced selective clearance of oligomeric betaamyloid peptide(1-42) by rat primary type 2 microglia. J Immunol 181: 6503-6513.

Staff NP, Lucchinetti CF, Keegan BM (2009). Multiple sclerosis with predominant, severe cognitive impairment. Arch Neurol 66: 1139-1143.

Terry RD (1996). The pathogenesis of Alzheimer disease: an alternative to the amyloid hypothesis. J Neuropathol Exp Neurol 55: 1023-1025.

Tiemann L, Penner IK, Haupts M, Schlegel U, Calabrese P (2009). Cognitive decline in multiple sclerosis: impact of topographic lesion distribution on differential cognitive deficit patterns. Mult Scler 15: 1164-1174.

Tombaugh TN (2006). A comprehensive review of the paced auditory serial addition test (PASAT). Arch Clin Neuropsychol 21: 53-76.

Townsend M, Qu Y, Gray A, Wu Z, Seto T, Hutton M et al (2010). Oral treatment with a \{gamma\}-secretase inhibitor improves long-term potentiation in a mouse model of Alzheimer's disease. J Pharmacol Exp Ther 333: 110-119.

Valls-Solé J, Pascual-Leone A, Wassermann EM, Hallett M (1992). Human motor evoked responses to paired transcranial magnetic stimuli. Electroencephalogr Clin Neurophysiol 85: 355-364.

Vemuri P, Wiste HJ, Weigand SD, Shaw KM, Trojanowski JQ, Weiner MW, et al, On behalf of the Alzheimer's Disease Neuroimaging Initiative (2009). MRI and CSF biomarkers in normal, MCI, and AD subjects. Diagnostic discrimination and cognitive correlations. Neurology 73: 287-293.

Walther T, Albrecht D, Becker M, Schubert M, Kouznetsova E, Wiesner B et al (2009). Improved learning and memory in aged mice deficient in amyloid beta-degrading neutral endopeptidase. PLoS One 4: e4590.

Zeller D, aufm Kampe K, Biller A, Stefan K, Gentner R, Schütz A et al (2010). Rapid-onset central motor plasticity in multiple sclerosis. Neurology 74: 728-735.

Ziemann U (2004). TMS induced plasticity in human cortex. Rev Neurosci 15: 253-266.

Ziemann U, Tergau F, Wischer S, Hildebrandt J, Paulus W (1998). Pharmacological control of facilitatory I-wave interaction in the human motor cortex. A paired transcranial magnetic stimulation study. Electroencephalogr Clin Neurophysiol 109: $321-330$.

Supplementary Information accompanies the paper on the Neuropsychopharmacology website (http://www.nature.com/npp) 